\title{
Quadratures with Remainders of Minimum Norm. I *
}

\section{By R. E. Barnhill and J. A. Wixom}

1. Introduction. One approach to the problem of numerical integration on contours in the complex plane was discussed in a previous paper [1]. This paper is an application of that work to the quadrature problem $\int_{-1}^{1} f(x) \cong \sum_{k=1}^{n} A_{k} f\left(z_{k}\right)$. For a fixed $n,\left\{A_{k}\right\}_{k=1}^{n}$ and $\left\{z_{k}\right\}_{k=1}^{n}$ form a set of $2 n$ variables. Let $R_{n}(f)=\int_{-1}^{1} f(z) d z-$ $\sum_{k=1}^{n} A_{k} f\left(z_{k}\right)$. Then $R_{n}$ is a linear functional defined on a certain function space $X$. In the cases to be discussed, $X$ is a Hilbert space. Suppose that $\left\{P_{m}\right\}_{m=0}^{\infty}$ is a closed set of functions in $X$. Many of the standard quadrature methods amount to specifying certain side conditions and, subject to these, choosing the $A_{k}$ and $z_{k}$ so that $R_{n}{ }^{-1}(0)=\left\{f \in X: R_{n}(f)=0\right\}$ contains a maximal number of the $P_{m}$. If $P_{0}, P_{1}, \cdots, P_{s}$ are in $R_{n}^{-1}(0)$ and $P_{s+1}$ is not in $R_{n}^{-1}(0)$, then the quadrature is said to have precision $s$. Thus, for example, if $X=C[a, b]$ and $P_{m}(x)=x^{m}$, $m=0,1, \cdots$, and the side conditions are that the $z_{k}$ are specified beforehand, then choosing the $A_{k}$ so that $R_{n}{ }^{-1}(0)$ contains a maximal number of the $P_{m}(x)$ amounts to the usual interpolatory quadrature procedure.

The procedure used in this paper is to minimize the norm of a certain error functional related to $R_{n}$. Specifically, suppose that $R_{n}(f)=R_{n}{ }^{*}\left(f^{(r)}\right)$, where $f^{(r)}(z)$ denotes the $r$ th derivative of $f$, and $R_{n}{ }^{*}$ is a linear functional defined on the space of $r$ th derivatives of functions in $X$. Then $\left|R_{n}(f)\right|=\left|R_{n}{ }^{*}\left(f^{(r)}\right)\right| \leqq\left\|R_{n}{ }^{*}\right\| \cdot\left\|f^{(r)}\right\|$, and the problem is that of minimizing $\left\|R_{n}{ }^{*}\right\|$ by an appropriate choice of the $A_{k}$ and $z_{k}$. It has been shown [1] that minimizing with respect to the $A_{k}$ (for fixed $z_{k}$ ) yields systems of linear equations to be solved; minimizing with respect to the $z_{k}$ yields systems of nonlinear equations. In this paper, the $z_{k}$ are assumed given beforehand and only the $A_{k}$ are treated as variables. In a later paper, both the linear and nonlinear cases will be considered.

2. The Equations to be Solved. In this paper we consider three sets of quadratures. For the first two sets, the space $X$ is $L^{2}\left(E_{\rho}\right)$, the $L^{2}$ completion of $H\left(E_{\rho}\right)$, where $H\left(E_{\rho}\right)=\left\{f: f\right.$ is analytic on the ellipse $E_{\rho}$ with semimajor axis $a$, semiminor axis $b=\left(a^{2}-1\right)^{1 / 2}$ and $\left.\rho=(a+b)^{2}\right\}$ [2]. The "double integral norm" is obtained from the inner product

$$
(f, g)=\iint_{E_{\rho}} f(z) \overline{g(z)} d x d y .
$$

For the first set of quadratures, $\left\{P_{m}(z)\right\}_{m=0}^{\infty}$ is defined by

$$
P_{m}(z)=2(m+1)^{1 / 2}\left[\pi\left(\rho^{m+1}-\rho^{-m-1}\right)\right]^{-1 / 2} U_{m}(z)
$$

Received February 21, 1966. Revised July 6, 1966.

* This research was supported in part by National Science Foundation Grant GP 2312 to the University of Utah Computer Center, Salt Lake City, Utah. 
where $U_{m}(z)=\left(1-z^{2}\right)^{-1 / 2} \sin [(m+1) \operatorname{arc} \cos (z)], m=0,1, \cdots$. In fact, the set $\left\{P_{m}(z)\right\}_{m=0}^{\infty}$ forms a closed orthonormal set in $L^{2}\left(E_{\rho}\right)$. No polynomial precision requirements are imposed for this set of quadratures so that $R_{n}=R_{n}{ }^{*}$ and $\left\|R_{n}\right\|^{2}=\sum_{m=0}^{\infty}\left|R_{n}\left(P_{m}\right)\right|^{2}$. If the $z_{k}$ are given in $[-1,1]$, then the $A_{k}$ are real and denoted by $a_{k}$. The resulting linear system of equations in the variables $a_{k}$ is as follows:

(1) $\sum_{j=1}^{n} a_{j}\left[\sum_{m=0}^{\infty} \alpha(m, \rho) U_{m}\left(z_{j}\right) U_{m}\left(z_{k}\right)\right]=\sum_{m=0}^{\infty} \alpha(m, \rho) \gamma(m) U_{m}\left(z_{k}\right), k=1, \cdots, n$, where $\alpha(m, \rho)=4(m+1)\left[\pi\left(\rho^{m+1}-\rho^{-m-1}\right)\right]^{-1}$, and

$$
\gamma(m)=(m+1)^{-1}\left[1+(-1)^{m}\right], \quad m=0,1, \cdots .
$$

In matrix notation, (1) can be written as $T a=h$, where $T=\left(t_{j k}\right), t_{j k}=$ $\sum_{m=0}^{\infty} \alpha(m, \rho) U_{m}\left(z_{j}\right) U_{m}\left(z_{k}\right), a=\left(a_{1}, \cdots, a_{n}\right)^{T}$, and $h=\left(h_{1}, h_{2}, \cdots, h_{n}\right)^{T}$ where $h_{k}=\sum_{m=0}^{\infty} \alpha(m, \rho) \gamma(m) U_{m}\left(z_{k}\right)$.

For the second set of quadratures, $\left\{P_{m}(z)\right\}_{m=0}^{\infty}$ is defined by $P_{m}{ }^{\prime}(z)=$ $2(m+1)^{1 / 2}\left[\pi\left(\rho^{m+1}-\rho^{-m-1}\right)\right]^{-1 / 2} U_{m}(z)$, where $U_{m}(z)$ is as above. For this set, precision is required for constant functions so that $\left\|R_{n}{ }^{*}\right\|^{2}=\sum_{m=0}^{\infty}\left|R_{n}\left(Q_{m}\right)\right|^{2}$, where $Q_{m}(z)=P_{m}^{\prime}(z)$. Assuming that the $z_{k}$ are given in $[-1,1]$, the resulting system of equations to be solved is the following:

$$
\begin{array}{r}
\sum_{j=1}^{n} a_{j}\left[2 \sum_{m=0}^{\infty} \alpha(m, \rho) T_{m+1}\left(z_{j}\right) T_{m+1}\left(z_{k}\right)\right]=2 \sum_{m=0}^{\infty} \alpha(m, \rho) \beta(m) T_{m+1}\left(z_{k}\right)-\lambda_{1}, \\
k=1, \cdots, n,
\end{array}
$$

where $\lambda_{1}$ is a parameter, $T_{m+1}(z)=\cos [(m+1) \operatorname{arc} \cos (z)]$, the $(m+1)$ st Tchebycheff polynomial of the first kind, $a_{j}$ and $\alpha(m, \rho)$ are as above, and $\beta(0)=0$, $\beta(m)=\left[1-(m+1)^{2}\right]^{-1}\left[1+(-1)^{m+1}\right], m=1,2, \cdots$. The system of equations (2) can be written in matrix form as $T a=h-\lambda$, where

$$
\begin{aligned}
T & =\left(t_{j k}\right), \\
t_{j k} & =2 \sum_{m=0}^{\infty} \alpha(m, \rho) T_{m+1}\left(z_{j}\right) T_{m+1}\left(z_{k}\right), \\
a & =\left(a_{1}, \cdots, a_{n}\right)^{T} \\
h & =\left(h_{1}, \cdots, h_{n}\right)^{T}, \\
h_{k} & =2 \sum_{m=0}^{\infty} \alpha(m, \rho) \beta(m) T_{m+1}\left(z_{k}\right),
\end{aligned}
$$

and

$$
\lambda=\left(\lambda_{1}, \lambda_{1}, \cdots, \lambda_{1}\right)^{T} .
$$

In the third set of quadratures, the space $X$ is the $L^{2}$ completion of $H([-1,1])$, where $H([-1,1])=\{f: f$ is analytic on $[-1,1]\}$. The "line integral norm" is obtained from the inner product

$$
(f, g)=\int_{-1}^{1}\left(1-z^{2}\right)^{1 / 2} f(z) \overline{g(z)}|d z|,
$$


where $|d z|$ denotes arc length. The $L^{2}$ completion of $H([-1,1])$ is with respect to the norm induced by this inner product. The Tchebycheff polynomials of the second kind form a closed orthogonal set in this space. Precision is required for constant functions so that $R_{n}(f)=R_{n}{ }^{*}\left(f^{\prime}\right)$ where $f^{\prime}$ is the derivative of $f$. This is the case $r=1$ in Section 1 . The resulting system of linear equations to be solved is the following:

$$
\sum_{j=1}^{n} a_{j}\left[2 \sum_{m=0}^{\infty} \delta(m) T_{m+1}\left(z_{j}\right) T_{m+1}\left(z_{k}\right)\right]=2 \sum_{m=0}^{\infty} \delta(m) \beta(m) T_{m+1}\left(z_{k}\right)-\lambda_{1},
$$

$$
k=1, \cdots, n,
$$

where $\beta(m)$ and $T_{m+1}(z)$ are defined above, $\delta(m)=(m+1)^{-2}[\lambda(m)]^{2}$, $\lambda(m)=2 \cdot 1 \cdot 3 \cdot 5 \cdots(2 m+1) / 2 \cdot 4 \cdots(2 m+2)$, and $\lambda_{1}$ is a parameter. As before, system (3) can be written in matrix form as $T a=h-\lambda_{1}$.

3. Description of the Computations. The elements of the matrix $T$ and the vector $h$ are infinite sums and hence must be approximated by finite sums. The approximating finite sums were obtained by fixing a positive integer $N$ and summing the series until $N$ consecutive partial sums were identical within the double precision range of the computer (16 digits). For the double integral norm, $N$ was fixed at 25 , and the number of terms in the approximating series varied from 1050 to 35 as $a$ varied from 1.0001 to 5.0. For the line integral norm, $N$ was fixed at 500 and the number of terms in the approximating series averaged about 350,000. The slow rate of convergence of the series for the line integral norm made it impractical to calculate $T$ using more than ten abscissas. Using the IBM 7044 it requires approximately 9 hours to compute and sum 10,000 terms of the series in the matrix $T$ for the 16 Gauss abscissas.

A direct method was used to calculate $T^{-1}$. Then Newton's Method was used to improve the calculated $T^{-1}$. If $D_{0}$ is the approximation to $T^{-1}$, calculated by a direct method, then $D_{1}=D_{0}\left(2 I-T D_{0}\right)$ is the first iterate, with error measured by $E_{1}=I-T D_{1}$. The iteration was terminated after $m$ steps when all the elements of $E_{m}$ were less than $10^{-14}$ in absolute value.

For the double integral norm calculations, the matrix $T$ becomes unstable with increasing values of $a$. For example, the determinant of $T$, using Gauss 7 point abscissas and $a \geqq 1.75$, becomes smaller than $10^{-27}$.

For this reason we were unable to obtain values for the quadrature weights and remainders with predictable reliability for some sets of base points for relatively large values of $a$. Hence, these are omitted from the following tables.

The linear system of equations is solved by the Gauss-Seidel method [4]. Since $T$ is real, positive definite, symmetric and has positive diagonal terms, the GaussSeidel method is known to converge.

The approximations to $\left\|R_{n}{ }^{*}\right\|$ and $\left\|R_{n}\right\|$ were calculated in the same manner as the approximations to the elements of the matrix $T$. Lee, Sun, and Lo [5] have calculated $\left\|R_{n}\right\|$ using known quadrature weights and we have verified their results as a check on our calculations.

4. Tables. The following set of tables (tables 1-j) lists the quadrature weights and the norms of the remainders that were calculated using known abscissas. These 
QUADRATURES WITH REMAINDERS OF MINIMUM NORM. I

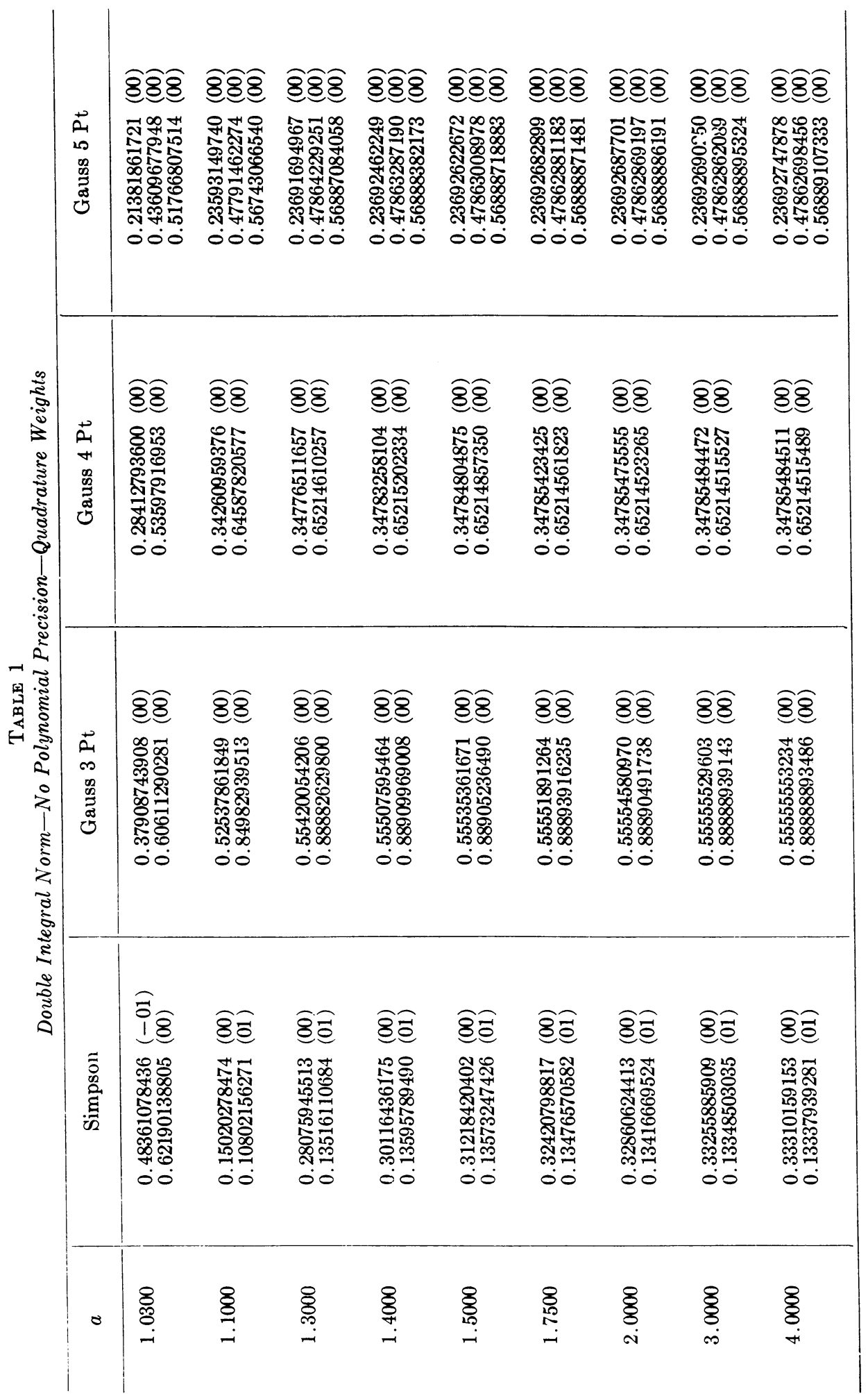




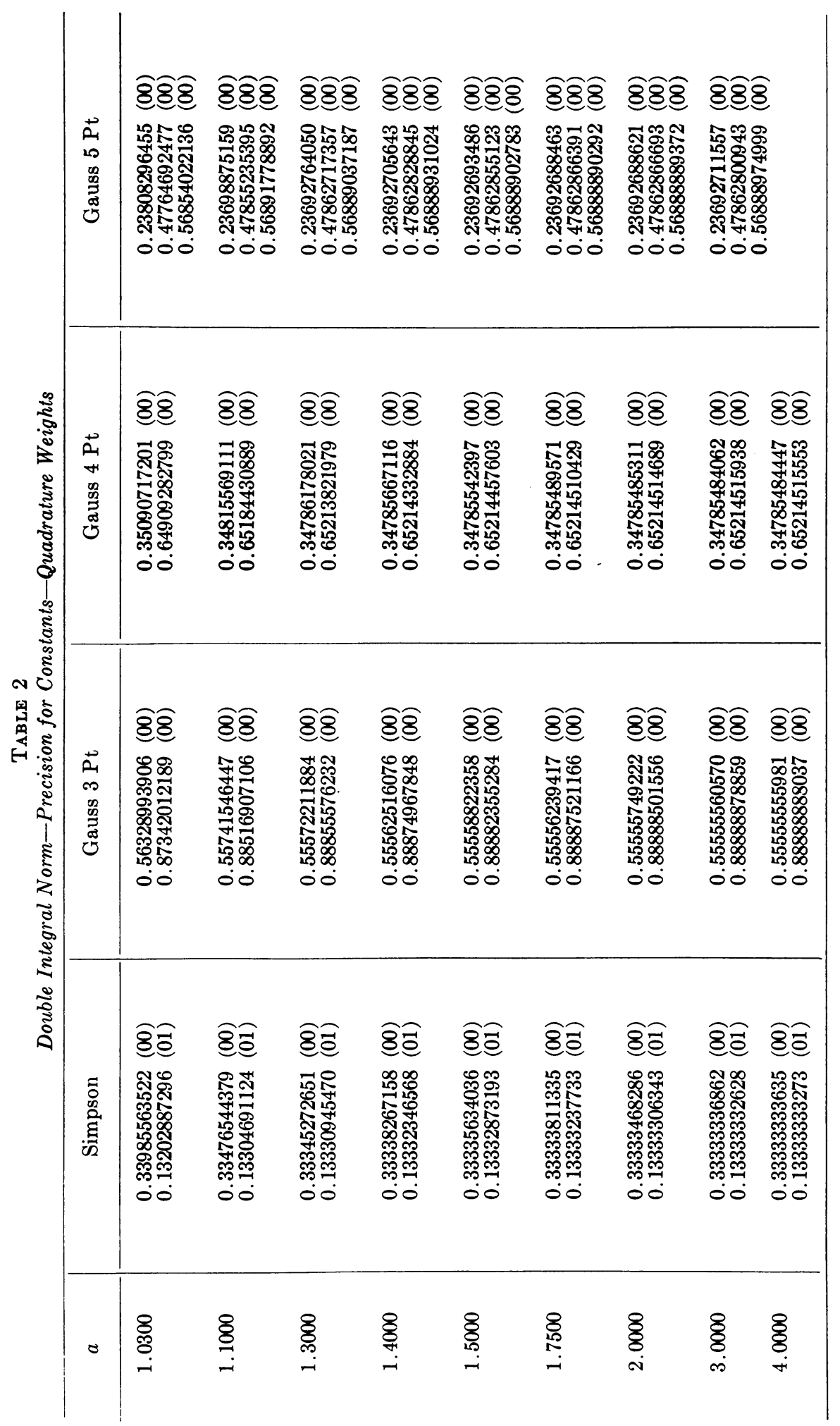



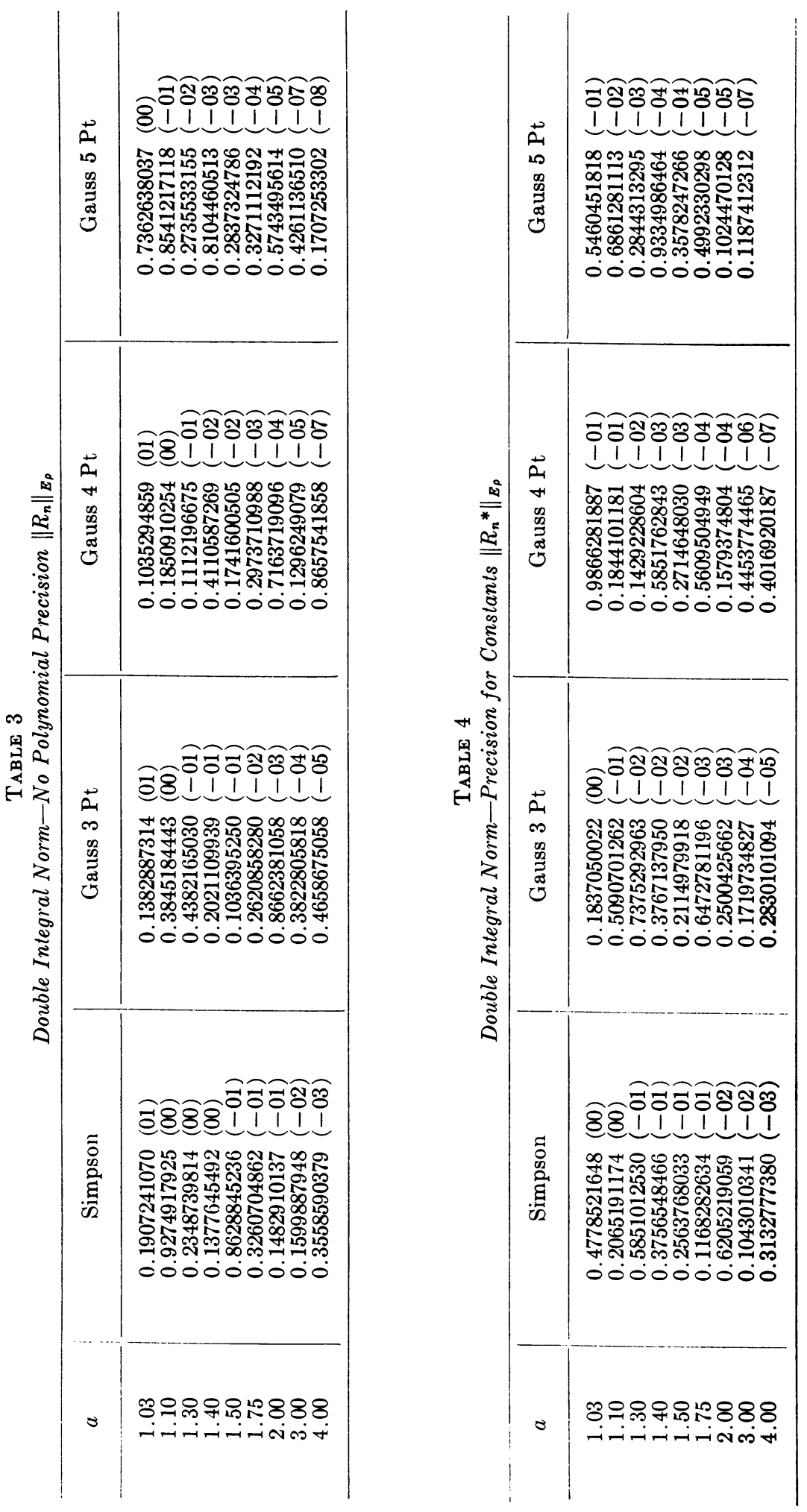
TABLE 5

Line Integral Norm-Precision for Constants

\begin{tabular}{|c|c|c|}
\hline Abscissas & Weights & $\left\|R_{n}^{*}\right\|$ \\
\hline Trapezoid & 1.00000000000 & 1.0650733423 \\
\hline Simpson & $\begin{array}{l}0.34166233122 \\
1.31667533756\end{array}$ & 0.3225321140 \\
\hline Weddle & $\begin{array}{l}0.11442868201 \\
0.40254092837 \\
0.31437632017 \\
0.33730813889\end{array}$ & 0.0681382406 \\
\hline Gauss 2 pt. & 1.00000000000 & 0.2550563770 \\
\hline Gauss $3 \mathrm{pt}$ & $\begin{array}{l}0.56573797607 \\
0.86852404786\end{array}$ & 0.1498311203 \\
\hline Gauss $4 \mathrm{pt}$ & $\begin{array}{l}0.35402340648 \\
0.64597659352\end{array}$ & 0.1020021252 \\
\hline Gauss $5 \mathrm{pt}$ & $\begin{array}{l}0.24103044858 \\
0.47512812123 \\
0.56768286040\end{array}$ & 0.0752714571 \\
\hline Gauss $7 \mathrm{pt}$ & $\begin{array}{l}0.13156595754 \\
0.27822895864 \\
0.38147838891 \\
0.41745338982\end{array}$ & 0.0471841912 \\
\hline Gauss $10 \mathrm{pt}$ & $\begin{array}{r}0.06074063246 \\
0.28496489128 \\
-0.48032018629 \\
1.60088303881 \\
-0.46626837611\end{array}$ & 0.3475518407 \\
\hline
\end{tabular}

quantities were calculated for 22 values of $a$ ranging from 1.0001 to 5.0 for abscissas corresponding to the following quadrature rules: trapezoid, Simpson, Gauss 2, 3, $4,5,7,10$, and 16 point rules, and Weddle. For the trapezoidal rule and the Gauss 2 -point rule, the weights are 1.0. The quantities in the above list that are not in the tables in this paper are in the Unpublished Mathematical Tables File.

The quadrature weights are labeled from left to right on $[-1,1]$. Since symmetric abscissas yield symmetric weights, the duplications are omitted from the tables. The numbers are expressed as floating point decimals with the number in parentheses being the power of ten.

5. Examples and Use of Tables. The table following the examples (table 6) gives comparisons, for specific functions, of our quadratures with various known quadratures. For the examples given, the same abscissas were used for both the known quadratures and our quadratures. The numbers in parenthesis under the double integral norms indicate the values of $a$ from which the quadrature weights were computed.

As a specific example for using the minimum norm remainders listed in the previous tables, consider the function $f(z)=e^{z^{2}}$.

(a) Double integral norm-no polynomial precision:

$\left|R_{n}(f)\right| \leqq\left\|R_{n}\right\| \cdot\|f\|$, where $\left\|R_{n}\right\|$ is tabulated in the tables and 


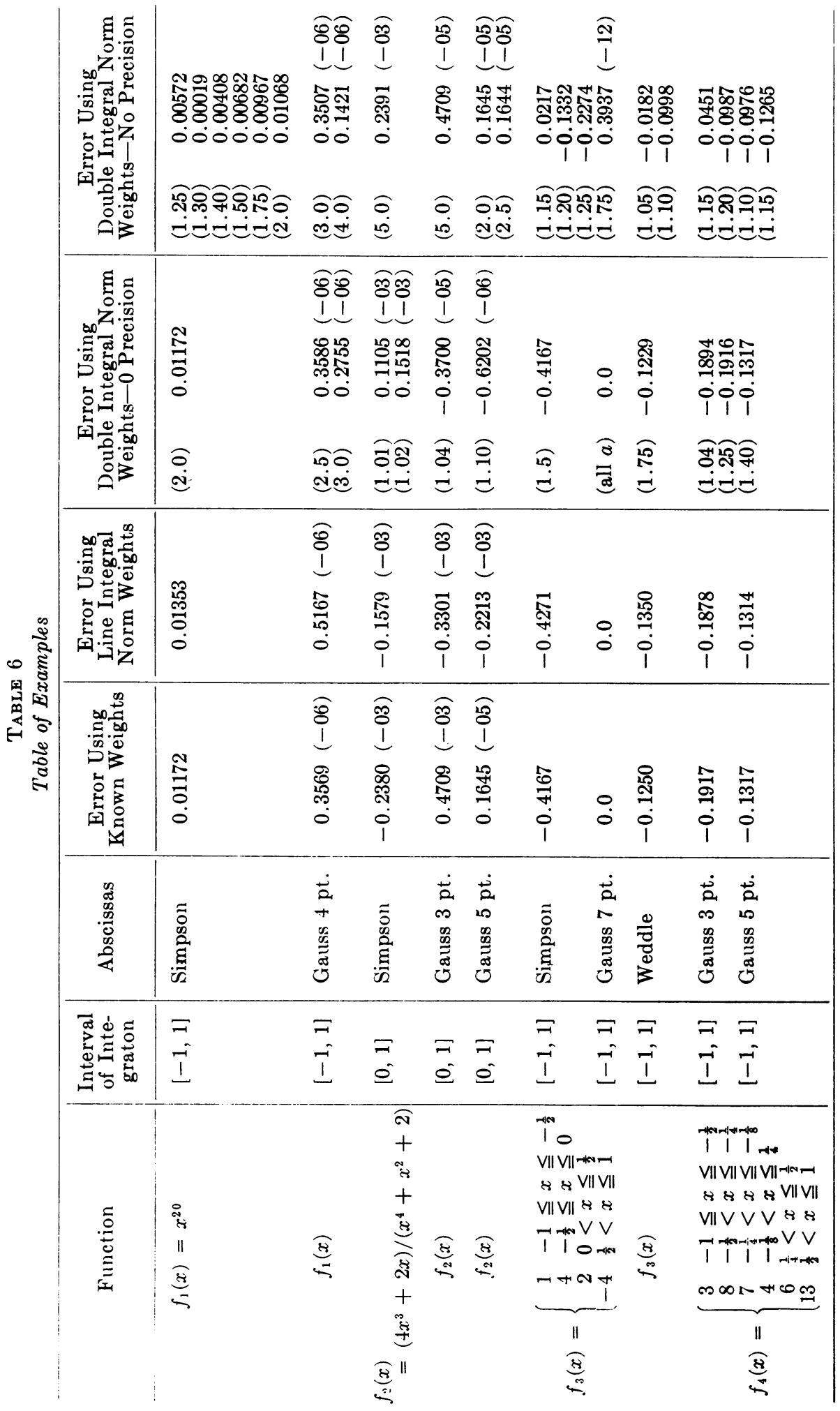




$$
\|f\|=\left\{\iint_{E_{\rho}}|f(z)|^{2} d x d y\right\}^{1 / 2} \leqq M\left\{\iint_{E_{\rho}} d x d y\right\}^{1 / 2}=M(\pi a b)^{1 / 2},
$$

where $M=\max _{z \in \boldsymbol{E}_{\rho}}|f(z)|$.

For $f(z)=e^{z^{2}}, M=\max _{z \in E_{\rho}}\left|e^{z^{2}}\right|=\max _{z \in E_{\rho}}\left|e^{x^{2}-y^{2}}\right|=e^{a^{2}}$.

Therefore, $\left|R_{n}(f)\right| \leqq\left\|R_{n}\right\| \cdot e^{a^{2}}(\pi a b)^{1 / 2}=\left\|R_{n}\right\| \cdot e^{a}\left[\pi a\left(a^{2}-1\right)^{1 / 2}\right]^{1 / 2}$.

The following minimum values of $\left\|R_{n}\right\| \cdot e^{a^{2}}(\pi a b)^{1 / 2}$ are obtained for the indicated abscissas from the values of $a$ used in the preceding tables.

Abscissas $\quad a \quad\left\|R_{n}\right\| \cdot e^{a^{2}}\left[\pi a\left(a^{2}-1\right)^{1 / 2}\right]^{1 / 2}$

Gauss 2 pt.

Simpson

Gauss 4 pt.

Gauss 7 pt.

Gauss 10 pt.
1.50

1.50

2.00

1.26993

1.87016

0.01290

$0.63077 \times 10^{-5}$

1.50

(b) Double integral norm-precision for constants:

$\left|R_{n}(f)\right| \leqq\left\|R_{n}{ }^{*}\right\| \cdot\left\|f^{\prime}\right\|$, where $\left\|R_{n}{ }^{*}\right\|$ is tabulated and

$$
\left\|f^{\prime}\right\|=\left\{\iint_{E_{\rho}}\left|f^{\prime}(z)\right|^{2} d x d y\right\}^{1 / 2} \leqq M^{\prime}(\pi a b)^{1 / 2}
$$

where $M^{\prime}=\max _{z \in E_{\rho}}\left|f^{\prime}(z)\right|$.

For $f(z)=e^{z^{2}}, M^{\prime}=\max _{z \in E_{\rho}}\left|2 z e^{z^{2}}\right|=\max _{z \in E_{\rho}} 2\left(x^{2}+y^{2}\right)^{1 / 2} e^{x^{2}-y^{2}}$, therefore, $M^{\prime}=2 a e^{a^{2}}$, and $\left\|f^{\prime}\right\| \leqq 2 a e^{a^{2}}(\pi a b)^{1 / 2}$.

$$
\left|R_{n}(f)\right| \leqq\left\|R_{n}^{*}\right\| \cdot 2 a e^{a^{2}}\left[\pi a\left(a^{2}-1\right)^{1 / 2}\right]^{1 / 2} .
$$

The following minimum values of $\left\|R_{n}{ }^{*}\right\| \cdot 2 a e^{a^{2}}\left[\pi a\left(a^{2}-1\right)^{1 / 2}\right]^{1 / 2}$ are obtained for the indicated abscissas from the values of $a$ used in the preceding tables.

Abscissas

Gauss 3 pt.

Simpson

Gauss 5 pt.

Weddle $a$

1.75

1.30

2.00

2.00
$\left\|R_{n}{ }^{*}\right\| \cdot 2 a e^{a^{2}}\left[\pi a\left(a^{2}-1\right)^{1 / 2}\right]^{1 / 2}$

\subsection{1}

1.51852

$0.73809 \times 10^{-3}$

$0.12802 \times 10^{-1}$

(c) Line integral norm-precision for constants:

$\left|R_{n}(f)\right| \leqq\left\|R_{n}{ }^{*}\right\| \cdot\left\|f^{\prime}\right\|$, where $\left\|R_{n}{ }^{*}\right\|$ is tabulated and

$$
\left\|f^{\prime}\right\|=\left\{\int_{-1}^{1}\left(1-z^{2}\right)^{1 / 2}\left|f^{\prime}(z)\right|^{2}|d z|\right\}^{1 / 2}
$$

where $|d z|$ is arc length.

$$
\left\|f^{\prime}\right\|=\left\{\int_{-1}^{1}\left(1-z^{2}\right)^{1 / 2}\left|f^{\prime}(z)\right|^{2}|d z|\right\}^{1 / 2}=\left\{\int_{-1}^{1}\left(1-x^{2}\right)^{1 / 2}\left|f^{\prime}(x)\right|^{2} d x\right\}^{1 / 2}
$$

for the contour $[-1,1]$. If we let $M=\max _{x \in[-1,1]}\left[\left(1-x^{2}\right)^{1 / 2}\left|f^{\prime}(x)\right|\right]^{2}$, then

$$
\left\|f^{\prime}\right\| \leqq \sqrt{ } M\left\{\int_{-1}^{1} d x\right\}^{1 / 2}=\sqrt{ } \overline{2 M}
$$




$$
\begin{aligned}
& \text { For } f(z)=e^{z^{2}}, \\
& \qquad \begin{aligned}
\left\|f^{\prime}(z)\right\| & =\left\{\int_{-1}^{1}\left(1-x^{2}\right)^{1 / 2}\left|2 z e^{z^{2}}\right|^{2} \mid d z\right\}^{1 / 2} \\
& =2\left\{\int_{-1}^{1}\left(1-x^{2}\right)^{1 / 2} x^{2} e^{2 x^{2}} d x\right\}^{1 / 2} \leqq 2 \sqrt{ } e^{2} \cdot \sqrt{ } 2=2 e \sqrt{ } 2 .
\end{aligned}
\end{aligned}
$$

Therefore,

$$
\left|R_{n}(f)\right| \leqq\left\|R_{n}^{*}\right\| \cdot 2 e \sqrt{ } 2 .
$$

The following values for $\left\|R_{n}{ }^{*}\right\| 2 e \sqrt{ } 2$ are obtained for the indicated abscissas.

$\begin{array}{lc}\text { Abscissa } & \left\|R_{n}{ }^{*}\right\| 2 e \sqrt{ } 2 \\ \text { Simpson } & 2.47978 \\ \text { Weddle } & 0.52388 \\ \text { Gauss } 3 \text { pt. } & 1.15197 \\ \text { Gauss 5 pt. } & 0.57872 \\ \text { Gauss 7 pt. } & 0.36277\end{array}$

6. Conclusions. It appears as if the double integral norm quadratures with no polynomial precision will be the most practical of the three sets of quadratures. The line integral norm quadratures involved series which converge very slowly, as noted in Section 3. Hence these quadratures would probably be too expensive to compute for most practical purposes.

For the examples calculated, the results of using the double integral norm quadratures with precision for constants did not seem significantly different from the results obtained using Gaussian quadratures. However, most of these comparisons were made using Gaussian abscissas and hence the Gaussian quadratures probably appear better than they might if compared with quadratures involving other abscissas.

It might be noted that if the $z_{k}$ are given, but are complex, then the system of equations to be solved doubles in size. Also, as mentioned above, it is planned to treat the $z_{k}$ as variables as well as the $A_{k}$.

7. Acknowledgments. The authors wish to thank the University of Utah Computer Center for their cooperation in the preparation of this paper.

Mathematics Department

University of Utah

Salt Lake City, Utah

1. R. E. Barnhill, "Complex quadratures with reminders of minimum norm," Numer. Math., v. 1, 1965, pp. 384-390.

2. P. J. DAvis, "Errors of numerical approximation for analytic functions," J. Rational Mech. Anal., v. 2, 1953, pp. 303-313. MR 14, 907.

3. P. J. DAvis, "Errors of numerical approximation for analytic functions," Survey of Numerical Analysis, McGraw-Hill, New York, 1962, pp. 468-484. MR 24 *B1766.

4. P. Davis \& P. RABINOwITZ, "On the estimation of quadrature errors for analy tic functions," MTAC, v. 8, 1954, pp. 193-203. MR 16, 404.

5. V. N. Faddeeva, Computational Methods of Linear Algebra, Dover, New York, 1959. MR 20 * 6777 .

6. Y. T. Lo, S. W. LEE, \& B. Sun, “On Davis' method of estimating quadrature errors," Math. Comp., v. 19, 1965, pp. 133-138. MR $31 * 1771$. 